QCD Evolution Workshop 2013

International Journal of Modern Physics: Conference Series Vol. 25 (2014) 1460023 (9 pages)

(C) The Authors

DOI: $10.1142 / \mathrm{S} 2010194514600234$

\title{
LONG-RANGE RAPIDITY CORRELATIONS IN HEAVY-LIGHT ION COLLISIONS*
}

\author{
YURI V. KOVCHEGOV ${ }^{\dagger}$ and DOUGLAS E. WERTEPNY ${ }^{\ddagger}$ \\ Department of Physics, The Ohio State University \\ Columbus, $\mathrm{OH}$ 43210, USA \\ †kovchegov.1@asc.ohio-state.edu \\ ${ }^{\ddagger}$ wertepny.1@osu.edu
}

Published 2 January 2014

\begin{abstract}
We study two-particle long-range rapidity correlations arising in the early stages of heavy ion collisions in the saturation/Color Glass Condensate framework, assuming for simplicity that one colliding nucleus is much larger than the other. We calculate the two-gluon production cross section while including all-order saturation effects in the heavy nucleus with the lowest-order rescattering in the lighter nucleus. We find four types of correlations in the two-gluon production cross section: (i) geometric correlations, (ii) HBT correlations accompanied by a back-to-back maximum, (iii) away-side correlations, and (iv) near-side azimuthal correlations which are long-range in rapidity. The geometric correlations (i) are due to the fact that nucleons are correlated by simply being confined within the same nucleus and may lead to long-range rapidity correlations for the produced particles without strong azimuthal angle dependence. Somewhat surprisingly, long-range rapidity correlations (iii) and (iv) have exactly the same amplitudes along with azimuthal and rapidity shapes: one centered around $\Delta \phi=\pi$ with the other one centered around $\Delta \phi=0$ (here $\Delta \phi$ is the azimuthal angle between the two produced gluons). We thus observe that the early-time CGC dynamics in nucleus-nucleus collisions generates azimuthal non-flow correlations which are qualitatively different from jet correlations by being long-range in rapidity. If strong enough, they have the potential of mimicking the elliptic (and higher-order even-harmonic) flow in the di-hadron correlators: one may need to take them into account in the experimental determination of the flow observables.
\end{abstract}

Keywords: Rapidity correlations; the ridge; parton saturation.

PACS numbers: $25.75 .-\mathrm{q}, 25.75 . \mathrm{Gz}, 12.38 . \mathrm{Bx}, 12.38 . \mathrm{Cy}$

\footnotetext{
*This is an Open Access article published by World Scientific Publishing Company. It is distributed under the terms of the Creative Commons Attribution 3.0 (CC-BY) License. Further distribution of this work is permitted, provided the original work is properly cited.

${ }^{\dagger}$ Speaker.
} 


\section{General Expression for Two-Gluon Production in Heavy-Light Ion Collisions}

This proceedings contribution is based on the paper [1].

Consider two-gluon production in a collision of a large heavy ion (target) with a lighter nucleus (projectile). The projectile is assumed to be smaller than the target, but still large enough a nucleus for the two gluons to be produced in collisions of different nucleons in the projectile with the target nucleus. Formally we assume that $A_{2} \gg A_{1} \gg 1$, where $A_{1}$ and $A_{2}$ are respectively the atomic numbers of the projectile and the target. The target was large enough for the saturation effects to be important, such that $\alpha_{s}^{2} A_{2}^{1 / 3} \sim 1$. The saturation effects in the projectile are taken at the lowest non-trivial order with $\alpha_{s}^{2} A_{1}^{1 / 3} \ll 1$ : for two-gluon correlations this corresponds to only two nucleons interacting in the projectile nucleus. In terms of momentum scales the regime of interest is $k_{T} \gtrsim Q_{s 1}$, with $k_{T}$ the transverse momentum of either one of the produced gluons $\left(k_{1}\right.$ or $\left.k_{2}\right)$ and $Q_{s 1}$ the saturation scale of the projectile, which is much smaller than the saturation scale of the target, $Q_{s 2} \gg Q_{s 1} \gg \Lambda_{Q C D}$. The approximation used in [1] corresponds to calculating the two-gluon production in nucleus-nucleus $(A+A)$ collisions in the McLerranVenugopalan (MV) model [2] while keeping interactions with the projectile nucleus to the lowest non-trivial order of two interacting nucleons. This setup may not directly describe the bulk of proton-nucleus $(p+A)$ collisions (also known as the dense-dilute collisions); however, our setup may be relevant for high-multiplicity $p+A$ collisions.

The diagrams contributing to the two-gluon production are shown in Figs. 1, 2 and 3. There the horizontal solid lines denote the two valence quarks in the two incoming nucleons. Vertical solid lines denote the final state cuts, with the two produced gluons labeled by crosses. Vertical dashed lines denote the interaction with the target, which, in the high energy regime considered, happens over much shorter time scale than the time associated with produced gluon emissions and absorptions. We label the contribution to the cross section coming from the diagrams in Fig. 1 as 'square', since it corresponds to a direct square of production amplitude, while the interference graphs in Figs. 2 and 3 will be referred to as 'crossed' diagrams.

The resulting two-gluon production cross section can be written as a sum of two terms corresponding to two different classes of diagrams in Fig. 1 and in Figs. 2 and 3 (labeled 'square' and 'crossed') [1],

$$
\frac{d \sigma}{d^{2} k_{1} d y_{1} d^{2} k_{2} d y_{2}}=\frac{d \sigma_{\text {square }}}{d^{2} k_{1} d y_{1} d^{2} k_{2} d y_{2}}+\frac{d \sigma_{\text {crossed }}}{d^{2} k_{1} d y_{1} d^{2} k_{2} d y_{2}},
$$

with

$$
\begin{aligned}
& \frac{d \sigma_{\text {square }}}{d^{2} k_{1} d y_{1} d^{2} k_{2} d y_{2}}=\frac{\alpha_{s}^{2} C_{F}^{2}}{16 \pi^{8}} \int d^{2} B d^{2} b_{1} d^{2} b_{2} T_{1}\left(\boldsymbol{B}-\boldsymbol{b}_{1}\right) T_{1}\left(\boldsymbol{B}-\boldsymbol{b}_{2}\right) d^{2} x_{1} d^{2} y_{1} d^{2} x_{2} d^{2} y_{2} \\
& \times e^{-i \boldsymbol{k}_{1} \cdot\left(\boldsymbol{x}_{1}-\boldsymbol{y}_{1}\right)-i \boldsymbol{k}_{2} \cdot\left(\boldsymbol{x}_{2}-\boldsymbol{y}_{2}\right)} \frac{\boldsymbol{x}_{1}-\boldsymbol{b}_{1}}{\left|\boldsymbol{x}_{1}-\boldsymbol{b}_{1}\right|^{2}} \cdot \frac{\boldsymbol{y}_{1}-\boldsymbol{b}_{1}}{\left|\boldsymbol{y}_{1}-\boldsymbol{b}_{1}\right|^{2}} \frac{\boldsymbol{x}_{2}-\boldsymbol{b}_{2}}{\left|\boldsymbol{x}_{2}-\boldsymbol{b}_{2}\right|^{2}} \cdot \frac{\boldsymbol{y}_{2}-\boldsymbol{b}_{2}}{\left|\boldsymbol{y}_{2}-\boldsymbol{b}_{2}\right|^{2}}
\end{aligned}
$$



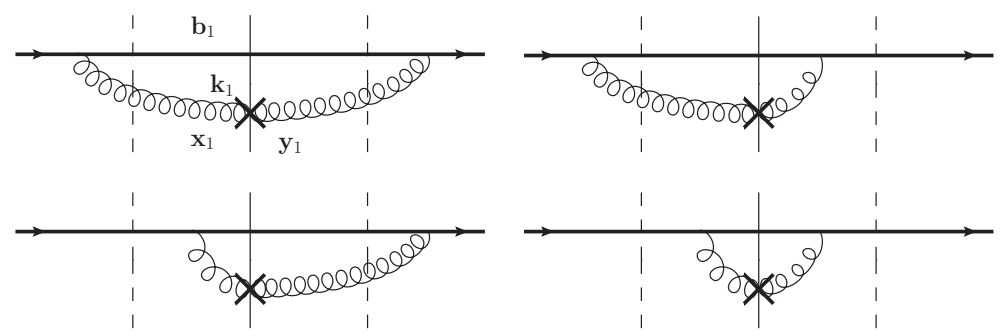

$\otimes$
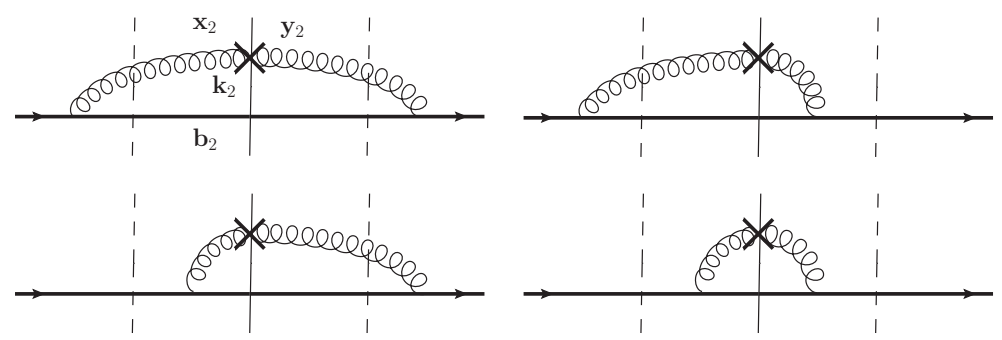

Fig. 1. Diagrams contributing to the two-gluon production cross section in the heavy-light ion collision. For clarity the diagrams are shown as a direct product of gluon production processes in collisions of the two interacting nucleons from the projectile nucleus with the target nucleus.
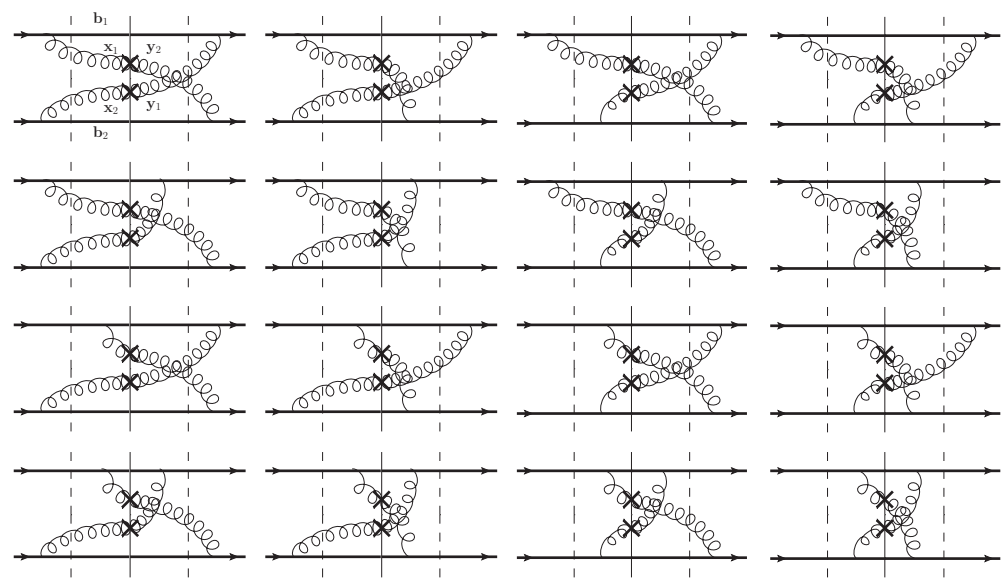

Fig. 2. Diagrams contributing to the two-gluon production cross section, with the gluon emitted by each nucleon in the amplitude absorbed by another nucleon in the complex conjugate amplitude. The top cross denotes the gluon with momentum $\boldsymbol{k}_{1}$, while the bottom one denotes the gluon with momentum $\boldsymbol{k}_{2}$. 

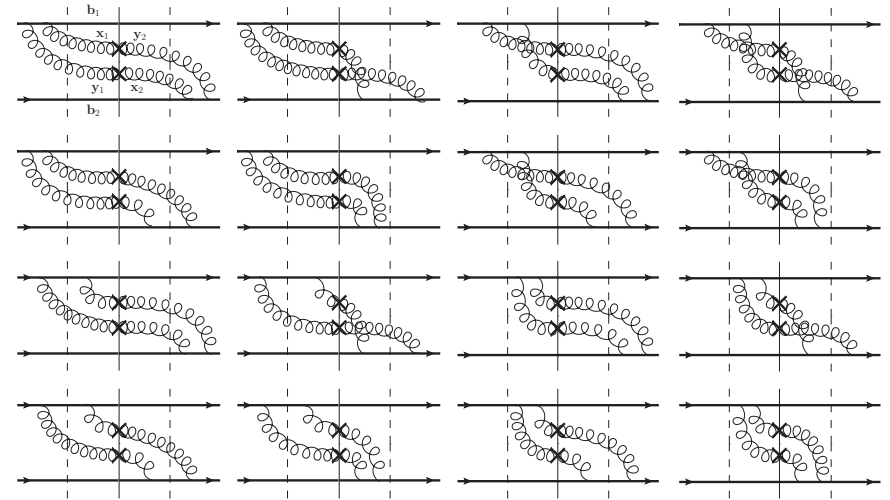

Fig. 3. Another set of diagrams contributing to the two-gluon production cross section, with the gluon emitted by each nucleon in the amplitude absorbed by another nucleon in the complex conjugate amplitude. Again the top cross denotes the gluon with momentum $\boldsymbol{k}_{1}$, while the bottom one denotes the gluon with momentum $\boldsymbol{k}_{2}$. Summation over the different orderings of gluon emissions (e.g. which gluon is emitted first or second) is implied but is not shown explicitly.

$$
\begin{aligned}
& \times\left\langle\left(\frac{1}{N_{c}^{2}-1} \operatorname{Tr}\left[U_{\boldsymbol{x}_{1}} U_{\boldsymbol{y}_{1}}^{\dagger}\right]-\frac{1}{N_{c}^{2}-1} \operatorname{Tr}\left[U_{\boldsymbol{x}_{1}} U_{\boldsymbol{b}_{1}}^{\dagger}\right]-\frac{1}{N_{c}^{2}-1} \operatorname{Tr}\left[U_{\boldsymbol{b}_{1}} U_{\boldsymbol{y}_{1}}^{\dagger}\right]+1\right)\right. \\
& \left.\times\left(\frac{1}{N_{c}^{2}-1} \operatorname{Tr}\left[U_{\boldsymbol{x}_{2}} U_{\boldsymbol{y}_{2}}^{\dagger}\right]-\frac{1}{N_{c}^{2}-1} \operatorname{Tr}\left[U_{\boldsymbol{x}_{2}} U_{\boldsymbol{b}_{2}}^{\dagger}\right]-\frac{1}{N_{c}^{2}-1} \operatorname{Tr}\left[U_{\boldsymbol{b}_{2}} U_{\boldsymbol{y}_{2}}^{\dagger}\right]+1\right)\right\rangle
\end{aligned}
$$

and

$$
\begin{aligned}
& \frac{d \sigma_{\text {crossed }}}{d^{2} k_{1} d y_{1} d^{2} k_{2} d y_{2}}=\int \frac{d^{2} B d^{2} b_{1} d^{2} b_{2}}{\left[2(2 \pi)^{3}\right]^{2}} T_{1}\left(\boldsymbol{B}-\boldsymbol{b}_{1}\right) T_{1}\left(\boldsymbol{B}-\boldsymbol{b}_{2}\right) d^{2} x_{1} d^{2} y_{1} d^{2} x_{2} d^{2} y_{2} \\
& \times\left[e^{-i \boldsymbol{k}_{1} \cdot\left(\boldsymbol{x}_{1}-\boldsymbol{y}_{2}\right)-i \boldsymbol{k}_{2} \cdot\left(\boldsymbol{x}_{2}-\boldsymbol{y}_{1}\right)}+e^{\left.-i \boldsymbol{k}_{1} \cdot\left(\boldsymbol{x}_{1}-\boldsymbol{y}_{2}\right)+i \boldsymbol{k}_{2} \cdot\left(\boldsymbol{x}_{2}-\boldsymbol{y}_{1}\right)\right]}\right. \\
& \times \frac{16 \alpha_{s}^{2}}{\pi^{2}} \frac{C_{F}}{2 N_{c}} \frac{\boldsymbol{x}_{1}-\boldsymbol{b}_{1}}{\left|\boldsymbol{x}_{1}-\boldsymbol{b}_{1}\right|^{2}} \cdot \frac{\boldsymbol{y}_{2}-\boldsymbol{b}_{2}}{\left|\boldsymbol{y}_{2}-\boldsymbol{b}_{2}\right|^{2}} \frac{\boldsymbol{x}_{2}-\boldsymbol{b}_{2}}{\left|\boldsymbol{x}_{2}-\boldsymbol{b}_{2}\right|^{2}} \cdot \frac{\boldsymbol{y}_{1}-\boldsymbol{b}_{1}}{\left|\boldsymbol{y}_{1}-\boldsymbol{b}_{1}\right|^{2}} \\
& \times\left[Q\left(\boldsymbol{x}_{1}, \boldsymbol{y}_{1}, \boldsymbol{x}_{2}, \boldsymbol{y}_{2}\right)-Q\left(\boldsymbol{x}_{1}, \boldsymbol{y}_{1}, \boldsymbol{x}_{2}, \boldsymbol{b}_{2}\right)-Q\left(\boldsymbol{x}_{1}, \boldsymbol{y}_{1}, \boldsymbol{b}_{2}, \boldsymbol{y}_{2}\right)+S_{G}\left(\boldsymbol{x}_{1}, \boldsymbol{y}_{1}\right)\right. \\
& -Q\left(\boldsymbol{x}_{1}, \boldsymbol{b}_{1}, \boldsymbol{x}_{2}, \boldsymbol{y}_{2}\right)+Q\left(\boldsymbol{x}_{1}, \boldsymbol{b}_{1}, \boldsymbol{x}_{2}, \boldsymbol{b}_{2}\right)+Q\left(\boldsymbol{x}_{1}, \boldsymbol{b}_{1}, \boldsymbol{b}_{2}, \boldsymbol{y}_{2}\right)-S_{G}\left(\boldsymbol{x}_{1}, \boldsymbol{b}_{1}\right) \\
& -Q\left(\boldsymbol{b}_{1}, \boldsymbol{y}_{1}, \boldsymbol{x}_{2}, \boldsymbol{y}_{2}\right)+Q\left(\boldsymbol{b}_{1}, \boldsymbol{y}_{1}, \boldsymbol{x}_{2}, \boldsymbol{b}_{2}\right)+Q\left(\boldsymbol{b}_{1}, \boldsymbol{y}_{1}, \boldsymbol{b}_{2}, \boldsymbol{y}_{2}\right)-S_{G}\left(\boldsymbol{b}_{1}, \boldsymbol{y}_{1}\right) \\
& \left.+S_{G}\left(\boldsymbol{x}_{2}, \boldsymbol{y}_{2}\right)-S_{G}\left(\boldsymbol{x}_{2}, \boldsymbol{b}_{2}\right)-S_{G}\left(\boldsymbol{b}_{2}, \boldsymbol{y}_{2}\right)+1\right] .
\end{aligned}
$$

We denote two-dimensional vectors in the transverse plane by $\boldsymbol{v}=\left(v^{x}, v^{y}\right)$ with their length $v_{T} \equiv|\boldsymbol{v}|$. As usual, $\alpha_{s}$ is the strong coupling constant, $N_{c}$ is the number of quark colors, and $C_{F}=\left(N_{c}^{2}-1\right) / 2 N_{c}$ is the Casimir operator of $\mathrm{SU}\left(N_{c}\right)$ in the fundamental representation. The center of the projectile nucleus is located at impact parameter $\boldsymbol{B}$ with respect to the center of the target nucleus, with $\boldsymbol{b}_{1}$ and $\boldsymbol{b}_{2}$ the impact parameters of the two interacting nucleons in the projectile, also measured with respect to the center of the target. The nuclear profile functions $T_{1}(\boldsymbol{b})$ describe 
the distribution of nucleons in the projectile. Angle brackets $\langle\ldots\rangle$ denote averaging in the target wave function squared.

The interactions with the target are described using

$$
U_{\boldsymbol{x}}=\mathrm{P} \exp \left\{i g \int_{-\infty}^{\infty} d x^{+} \mathcal{A}^{-}\left(x^{+}, x^{-}=0, \boldsymbol{x}\right)\right\},
$$

which is the adjoint Wilson line taken along the $x^{+}$light cone with $\mathcal{A}^{-}$the gluon field of the target nucleus in the adjoint representation. The contribution (3) depends on the $S$-matrices for the adjoint color-dipole

$$
S_{G}\left(\boldsymbol{x}_{1}, \boldsymbol{x}_{2}\right) \equiv \frac{1}{N_{c}^{2}-1}\left\langle\operatorname{Tr}\left[U_{\boldsymbol{x}_{1}} U_{\boldsymbol{x}_{2}}^{\dagger}\right]\right\rangle
$$

and adjoint color-quadrupole

$$
Q\left(\boldsymbol{x}_{1}, \boldsymbol{x}_{2}, \boldsymbol{x}_{3}, \boldsymbol{x}_{4}\right) \equiv \frac{1}{N_{c}^{2}-1}\left\langle\operatorname{Tr}\left[U_{\boldsymbol{x}_{1}} U_{\boldsymbol{x}_{2}}^{\dagger} U_{\boldsymbol{x}_{3}} U_{\boldsymbol{x}_{4}}^{\dagger}\right]\right\rangle
$$

We impose no ordering on the rapidities $y_{1}$ and $y_{2}$ of the two produced gluons. (We do assume that $\left|y_{1}-y_{2}\right| \ll 1 / \alpha_{s}$ and $0<Y-y_{1,2} \ll 1 / \alpha_{s}$, with $Y$ the rapidity of the projectile, such that no small- $x$ evolution corrections need to be included in the rapidity intervals between the gluons and between the projectile and the gluons.) In the case of rapidity-ordered two-gluon production (say, $y_{2} \gg y_{1}$ ) the corresponding cross section was found previously in [3], in apparent agreement with our Eq. (2) (modulo the nuclear profile functions $T_{1}(\boldsymbol{b})$ we included in the projectile nucleus). An expression for two-gluon production without rapidity ordering containing both the double-trace and quadrupole structures of Eqs. (2) and (3) was obtained in [4] shortly before our work [1].

\section{Matrix Elements of Wilson Lines in the Quasi-Classical Approximation}

The matrix elements of the double-trace, dipole and quadrupole operators entering Eqs. (2) and (3) can be evaluated using the Gaussian approximation (the MV model). When using this approximation one treats both the projectile and the target in the same consistent way, including only multiple interactions with target and projectile nucleons in the cross section. The drawback is, of course, that the resulting two-gluon production cross section is energy- and rapidity-independent, just like all other observables in the quasi-classical approximation. Inclusion of the full energy and rapidity dependence goes beyond the scope of the present work. However, evolution corrections can be readily included in the rapidity interval between the produced gluons and the target by evolving the double-trace, dipole and quadrupole operators using the BK $[5,6,7,8]$ and JIMWLK $[9,10]$ evolution equations.

In the MV model and in the large- $N_{c}$ approximation the double-trace, dipole and quadrupole operators entering Eqs. (2) and (3) were found in [1]. The results 
are as follows. For the double-trace operator we write

$$
\begin{array}{r}
\frac{1}{\left(N_{c}^{2}-1\right)^{2}}\left\langle\operatorname{Tr}\left[U_{\boldsymbol{x}_{1}} U_{\boldsymbol{x}_{2}}^{\dagger}\right] \operatorname{Tr}\left[U_{\boldsymbol{x}_{3}} U_{\boldsymbol{x}_{4}}^{\dagger}\right]\right\rangle=\frac{1}{\left(N_{c}^{2}-1\right)^{2}}\left\langle\operatorname{Tr}\left[U_{\boldsymbol{x}_{1}} U_{\boldsymbol{x}_{2}}^{\dagger}\right]\right\rangle\left\langle\operatorname{Tr}\left[U_{\boldsymbol{x}_{3}} U_{\boldsymbol{x}_{4}}^{\dagger}\right]\right\rangle \\
+\Delta\left(\boldsymbol{x}_{1}, \boldsymbol{x}_{2}, \boldsymbol{x}_{3}, \boldsymbol{x}_{4}\right) \\
+
\end{array}
$$

where $\Delta$ represents the subleading in $\frac{1}{N_{c}^{2}}$ terms in the matrix element. To leading order in $\frac{1}{N_{c}^{2}}$ in the MV model it is given by

$$
\begin{aligned}
\Delta\left(\boldsymbol{x}_{1}, \boldsymbol{x}_{2}, \boldsymbol{x}_{3}, \boldsymbol{x}_{4}\right) & =\frac{\left(D_{3}-D_{2}\right)^{2}}{N_{c}^{2}}\left[\frac{e^{D_{1}}}{D_{1}-D_{2}}-\frac{2 e^{D_{1}}}{\left(D_{1}-D_{2}\right)^{2}}+\frac{e^{D_{1}}}{D_{1}-D_{3}}\right. \\
& \left.-\frac{2 e^{D_{1}}}{\left(D_{1}-D_{3}\right)^{2}}+\frac{2 e^{\frac{1}{2}\left(D_{1}+D_{2}\right)}}{\left(D_{1}-D_{2}\right)^{2}}+\frac{2 e^{\frac{1}{2}\left(D_{1}+D_{3}\right)}}{\left(D_{1}-D_{3}\right)^{2}}\right]+O\left(\frac{1}{N_{c}^{4}}\right),
\end{aligned}
$$

where we have defined

$$
\begin{aligned}
& D_{1}=-\frac{Q_{s 2}^{2}}{4}\left[\left|\boldsymbol{x}_{1}-\boldsymbol{x}_{2}\right|^{2} \ln \left(\frac{1}{\left|\boldsymbol{x}_{1}-\boldsymbol{x}_{2}\right| \Lambda}\right)+\left|\boldsymbol{x}_{3}-\boldsymbol{x}_{4}\right|^{2} \ln \left(\frac{1}{\left|\boldsymbol{x}_{3}-\boldsymbol{x}_{4}\right| \Lambda}\right)\right] \\
& D_{2}=-\frac{Q_{s 2}^{2}}{4}\left[\left|\boldsymbol{x}_{1}-\boldsymbol{x}_{3}\right|^{2} \ln \left(\frac{1}{\left|\boldsymbol{x}_{1}-\boldsymbol{x}_{3}\right| \Lambda}\right)+\left|\boldsymbol{x}_{2}-\boldsymbol{x}_{4}\right|^{2} \ln \left(\frac{1}{\left|\boldsymbol{x}_{2}-\boldsymbol{x}_{4}\right| \Lambda}\right)\right] \\
& D_{3}=-\frac{Q_{s 2}^{2}}{4}\left[\left|\boldsymbol{x}_{1}-\boldsymbol{x}_{4}\right|^{2} \ln \left(\frac{1}{\left|\boldsymbol{x}_{1}-\boldsymbol{x}_{4}\right| \Lambda}\right)+\left|\boldsymbol{x}_{2}-\boldsymbol{x}_{3}\right|^{2} \ln \left(\frac{1}{\left|\boldsymbol{x}_{2}-\boldsymbol{x}_{3}\right| \Lambda}\right)\right]
\end{aligned}
$$

with $Q_{s 2}$ the target saturation scale for gluons in the quasi-classical (MV) limit and $\Lambda$ an infrared (IR) cutoff.

The $S$-matrix $S_{G}$ for the gluon color dipole interaction with the target and in the MV model is

$$
S_{G}\left(\boldsymbol{x}_{1}, \boldsymbol{x}_{2}\right)=\exp \left[-\frac{1}{4}\left|\boldsymbol{x}_{1}-\boldsymbol{x}_{2}\right|^{2} Q_{s 2}^{2}\left(\frac{\boldsymbol{x}_{1}+\boldsymbol{x}_{2}}{2}\right) \ln \left(\frac{1}{\left|\boldsymbol{x}_{1}-\boldsymbol{x}_{2}\right| \Lambda}\right)\right]
$$

The gluon color-quadrupole $S$-matrix in the MV model and in the large- $N_{c}$ approximation is

$$
Q\left(\boldsymbol{x}_{1}, \boldsymbol{x}_{2}, \boldsymbol{x}_{3}, \boldsymbol{x}_{4}\right)=\left[e^{D_{1} / 2}+\frac{D_{3}-D_{2}}{D_{1}-D_{3}}\left(e^{D_{1} / 2}-e^{D_{3} / 2}\right)\right]^{2} .
$$

These results, when used in Eqs. (1), (2) and (3), give one the expression for the two-gluon production cross section in heavy-light ion collisions in the quasi-classical approximation of the MV model.

\section{Correlation Function}

To study the properties of the obtained two-gluon production cross section, let us define the correlation function by

$$
C\left(\boldsymbol{k}_{1}, y_{1}, \boldsymbol{k}_{2}, y_{2}\right)=\mathcal{N} \frac{\frac{d \sigma}{d^{2} k_{1} d y_{1} d^{2} k_{2} d y_{2}}}{\frac{d \sigma}{d^{2} k_{1} d y_{1}} \frac{d \sigma}{d^{2} k_{2} d y_{2}}}-1
$$


where the normalization factor $\mathcal{N}$ is usually fixed by requiring that the numerator of $C$ (after reducing both terms in (12) to the common denominator) integrates out to zero when integrating over the whole sample defined by the cuts. For instance, the correlator as a function of gluon rapidities $y_{1}, y_{2}$ and azimuthal angles $\phi_{1}, \phi_{2}$ for gluons with fixed magnitudes of their transverse momenta $k_{1}, k_{2}$ is given by

$$
C\left(\boldsymbol{k}_{1}, y_{1}, \boldsymbol{k}_{2}, y_{2}\right)=\frac{\left[\int d \phi_{1} d y_{1} \frac{d \sigma}{d^{2} k_{1} d y_{1}} \int d \phi_{2} d y_{2} \frac{d \sigma}{d^{2} k_{2} d y_{2}}\right]}{\left[\int d \phi_{1} d y_{1} d \phi_{2} d y_{2} \frac{d \sigma}{d^{2} k_{1} d y_{1} d^{2} k_{2} d y_{2}}\right]} \frac{\frac{d \sigma}{d^{2} k_{1} d y_{1} d^{2} k_{2} d y_{2}}}{\frac{d \sigma}{d^{2} k_{1} d y_{1}} \frac{d \sigma}{d^{2} k_{2} d y_{2}}}-1
$$

Using the properties of the adjoint Wilson lines one can show that $\operatorname{Tr}\left[U_{\boldsymbol{x}} U_{\boldsymbol{y}}^{\dagger}\right]=$ $\operatorname{Tr}\left[U_{\boldsymbol{y}} U_{\boldsymbol{x}}^{\dagger}\right]$ and $\operatorname{Tr}\left[U_{\boldsymbol{x}_{1}} U_{\boldsymbol{y}_{1}}^{\dagger} U_{\boldsymbol{x}_{2}} U_{\boldsymbol{y}_{2}}^{\dagger}\right]=\operatorname{Tr}\left[U_{\boldsymbol{y}_{2}} U_{\boldsymbol{x}_{2}}^{\dagger} U_{\boldsymbol{y}_{1}} U_{\boldsymbol{x}_{1}}^{\dagger}\right]$. When these relations are used in Eqs. (2) and (3) one can conclude that these two-gluon production cross sections are invariant under $\boldsymbol{k}_{1} \leftrightarrow \boldsymbol{k}_{2}$ and $\boldsymbol{k}_{2} \rightarrow-\boldsymbol{k}_{2}$ interchanges. This implies that the correlation function contains only even Fourier harmonics in its Fourier decomposition over $\Delta \phi=\phi_{1}-\phi_{2}$,

$$
\left.C\left(\boldsymbol{k}_{1}, y_{1}, \boldsymbol{k}_{2}, y_{2}\right)\right|_{A_{2} \gg A_{1} \gg 1} \sim \sum_{n=0}^{\infty} d_{n}\left(k_{1 T}, k_{2 T}\right) \cos (2 n \Delta \phi),
$$

with some coefficients $d_{n}$. Therefore the near- and away-side correlations (that is, correlations around $\Delta \phi=0$ and $\Delta \phi=\pi$ respectively) resulting from this calculation are identical. A sample correlation is illustrated in Fig. 4. The correlations are also flat in rapidity up to $\left|y_{1}-y_{2}\right| \lesssim 1 / \alpha_{s}$, making them a plausible contributor to the ridge correlation observed in $A+A, p+A$ and $p+p$ collisions at RHIC and LHC.

As one can show [1], at the lowest order in multiple rescatterings the cross sections in Eqs. (2) and (3) yield the contribution studied originally in [11, 12] (see also [13]). The examples of the corresponding lowest-order diagrams are shown in Fig. 5.

It is interesting to note that the diagram in the right panel of Fig. 5 gives the two-gluon production cross section proportional to the Fourier transform of the

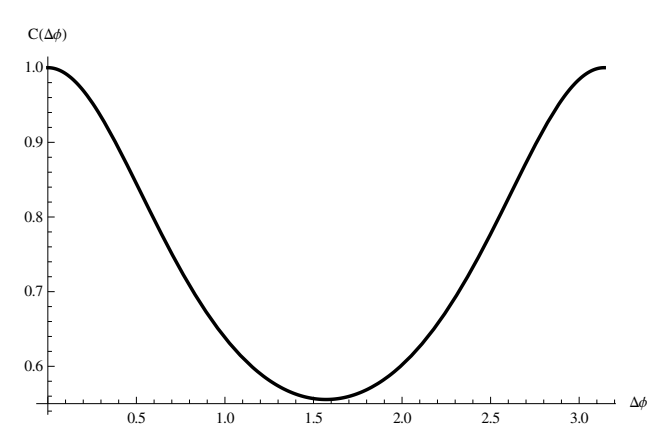

Fig. 4. A toy azimuthal two-gluon correlation function motivated by this calculation. Vertical scale is arbitrary. 

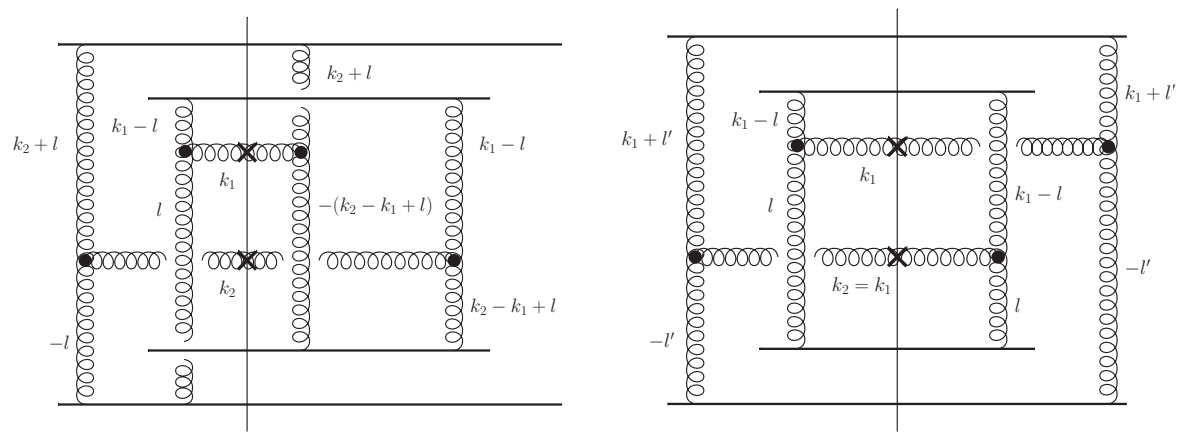

Fig. 5. Examples of diagrams generating contributions to correlated two-gluon production at the lowest non-trivial order (cf. [11]). The $t$-channel gluon momenta flow toward the triple-gluon vertices to the left of the cut, and away from those vertices to the right of the cut.

overlap region of the nuclear profile functions of the two nuclei, $T_{1}$ and $T_{2}$,

$$
\begin{array}{r}
\frac{d \sigma_{H B T}}{d^{2} k_{1} d y_{1} d^{2} k_{2} d y_{2}} \sim \int d^{2} b_{1} d^{2} b_{2} T_{1}\left(\boldsymbol{B}-\boldsymbol{b}_{1}\right) T_{1}\left(\boldsymbol{B}-\boldsymbol{b}_{2}\right) T_{2}\left(\boldsymbol{b}_{1}\right) T_{2}\left(\boldsymbol{b}_{2}\right) \\
\times e^{-i\left(\boldsymbol{k}_{1}-\boldsymbol{k}_{2}\right) \cdot\left(\boldsymbol{b}_{1}-\boldsymbol{b}_{2}\right)}+\left(\boldsymbol{k}_{2} \rightarrow-\boldsymbol{k}_{2}\right) .
\end{array}
$$

These correlations have the trademark form of the Hanbury Brown-Twiss (HBT) correlations ${ }^{14}$ which are widely studied in heavy ion physics. The correlation resulting from Eq. (15) is a sharp peak at $\boldsymbol{k}_{1} \approx \boldsymbol{k}_{2}$, with its width inversely proportional to the size of the overlap region. (The longitudinal HBT correlation is absent since, due to the extreme Lorentz-contraction of the colliding nuclei, the overlap region giving rise to the initial-state dynamics studied here is very thin in the longitudinal direction.) Note that the second term on the right of Eq. (15) corresponds to the back-to-back HBT-like correlation at $\boldsymbol{k}_{1} \approx-\boldsymbol{k}_{2}$ : this is the new prediction of the saturation approach, to be tested experimentally in the future.

\section{Geometric Correlations}

There is another potentially important correlation contained in the two-gluon production cross section from Eqs. (2) and (3). To see it let us step back from our full calculation above and consider a general form for the single- and double-gluon production cross sections in the heavy-light ion collision. One can write

$$
\begin{aligned}
\frac{d \sigma}{d^{2} k d y} & =\int d^{2} B d^{2} b\left|\Psi_{I}(\boldsymbol{B}-\boldsymbol{b})\right|^{2}\left\langle\frac{d \sigma^{p A_{2}}}{d^{2} k d y d^{2} b}\right\rangle \\
\frac{d \sigma}{d^{2} k_{1} d y_{1} d^{2} k_{2} d y_{2}} & =\int d^{2} B d^{2} b_{1} d^{2} b_{2}\left|\Psi_{I I}\left(\boldsymbol{B}-\boldsymbol{b}_{1}, \boldsymbol{B}-\boldsymbol{b}_{2}\right)\right|^{2} \\
& \times\left\langle\frac{d \sigma^{p A_{2}}}{d^{2} k_{1} d y_{1} d^{2} b_{1}} \frac{d \sigma^{p A_{2}}}{d^{2} k_{2} d y_{2} d^{2} b_{2}}\right\rangle
\end{aligned}
$$

where $\Psi_{I}(\boldsymbol{b})$ and $\Psi_{I I}\left(\boldsymbol{b}_{1}, \boldsymbol{b}_{2}\right)$ are the 1- and 2-nucleon wave functions of the projectile nucleus. 
Analyzing Eqs. (16) we see that even if the two-gluon production in the scattering of two nucleons from the projectile on the target is uncorrelated for fixed impact parameters,

$$
\left\langle\frac{d \sigma^{p A_{2}}}{d^{2} k_{1} d y_{1} d^{2} b_{1}} \frac{d \sigma^{p A_{2}}}{d^{2} k_{2} d y_{2} d^{2} b_{2}}\right\rangle \approx\left\langle\frac{d \sigma^{p A_{2}}}{d^{2} k_{1} d y_{1} d^{2} b_{1}}\right\rangle\left\langle\frac{d \sigma^{p A_{2}}}{d^{2} k_{2} d y_{2} d^{2} b_{2}}\right\rangle,
$$

due to the $\boldsymbol{B}$-integral in Eq. (16b) this does not imply that gluon production is factorized after all impact parameters have been included,

$$
\frac{d \sigma^{p A_{2}}}{d^{2} k_{1} d y_{1} d^{2} k_{2} d y_{2}} \nsim \frac{d \sigma^{p A_{2}}}{d^{2} k_{1} d y_{1}} \frac{d \sigma^{p A_{2}}}{d^{2} k_{2} d y_{2}} .
$$

Therefore, the integration over the impact parameter $\boldsymbol{B}$ between the projectile and the target nuclei, even constrained to a given centrality bin, generates a correlation. We referred to this effect as a geometric correlation [1]. Indeed if both the magnitude $B$ and direction $\hat{B}$ (with respect to directions of the outgoing gluons momenta $\boldsymbol{k}_{1}$ and $\boldsymbol{k}_{2}$ ) of the impact parameter are fixed, then geometric correlations would disappear: however, fixing the vector $\boldsymbol{B}$ precisely may appear to be difficult in the analysis of the experimental data, especially in $p+A$ and $p+p$ collisions.

\section{Acknowledgments}

This research is sponsored in part by the U.S. Department of Energy under Grant No. DE-SC0004286.

\section{References}

1. Y. V. Kovchegov and D. E. Wertepny, Nucl.Phys. A906, 50-83 (2013).

2. L. D. McLerran and R. Venugopalan, Phys. Rev. D50, 2225-2233 (1994).

3. A. Kovner and M. Lublinsky, Phys.Rev. D83, 034017 (2011).

4. A. Kovner and M. Lublinsky, Int.J.Mod.Phys. E22, 1330001 (2013).

5. I. Balitsky, Nucl. Phys. B463, 99-160 (1996).

6. I. Balitsky, Phys. Rev. D60, 014020 (1999).

7. Y. V. Kovchegov, Phys. Rev. D60, 034008 (1999).

8. Y. V. Kovchegov, Phys. Rev. D61, 074018 (2000).

9. J. Jalilian-Marian, A. Kovner, and H. Weigert, Phys. Rev. D59, 014015 (1998).

10. E. Iancu, A. Leonidov, and L. D. McLerran, Nucl. Phys. A692 , 583-645 (2001).

11. A. Dumitru, F. Gelis, L. McLerran, and R. Venugopalan, Nucl. Phys. A810, 91-108 (2008).

12. K. Dusling, F. Gelis, T. Lappi, and R. Venugopalan, Nucl. Phys. A836, 159-182 (2010).

13. S. Gavin, L. McLerran, and G. Moschelli, Phys. Rev. 051902, C79 (2009).

14. R. Hanbury Brown and R. Twiss, Nature 178, 1046-1048 (1956). 